\title{
Association between Coffee Consumption and Renal Impairment in Korean Women with and without Diabetes: Analysis of the Fourth Korea National Health and Nutrition Examination Survey in 2008

\section{Bo Ha Kim, Yong Soon Park ${ }^{1}$, Hye Mi Noh², Ji Sun Sung ${ }^{3}$, Jung Kwon Lee,}

Department of Family Medicine, Kangbuk Samsung Hospital, SungKyunKwan University, School of Medicine, Seoul; ${ }^{1}$ Department of Family Medicine, Hallym University Chuncheon Sacred Heart Hospital, Chuncheon; ${ }^{2}$ Department of Family Medicine, Hallym University Sacred Heart Hospital, Anyang; ${ }^{3}$ Health Screening Center, Kangbuk Samsung Hospital, Sungkyunkwan University School of Medicine, Suwon; ${ }^{4}$ Department of Family Medicine, Samsung Medical Center, Sungkyunkwan University School of Medicine, Seoul, Korea

Background: Recent studies suggest that coffee consumption has an influence on kidney function. This study investigated the relationship between habitual coffee consumption and renal impairment in Korean women, in consideration of diabetic status.

Methods: This study involved 2,673 women aged 35 to 84 years who had participated in the Fourth Korea National Health and Nutrition Examination Surveys, conducted in 2008. Habitual coffee consumption was classified into three categories: less than 1 cup per day, 1 cup per day, and 2 or more cups per day. Renal function impairment was defined as an estimated glomerular filtration rate less than $60 \mathrm{~mL} / \mathrm{min} / 1.73 \mathrm{~m}^{2}$ by the Modification of Diet in Renal Disease equation.

Results: The prevalence of diabetes and renal function impairment was higher in women who drank $<1$ cup of coffee per day. Compared with drinking < 1 cup of coffee per day, the odds ratio (OR) for renal function impairment was significantly lower (OR, 0.59; 95\% confidence interval [CI], 0.37 to $0.95 ; \mathrm{P}=0.03$ ) in those who habitually drank $\geq 2$ cups per day after adjusting for multiple confounding factors. When data were stratified according to the presence of diabetes, coffee consumption $\geq 2$ cups of coffee per day showed an inverse association with renal function impairment in only diabetic women (OR, $0.14 ; 95 \%$ CI, 0.02 to 0.88 ; $\mathrm{P}=0.04$ ), compared with consumption < 1 cup of coffee per day.

Conclusion: In a representative sample of Korean women, coffee consumption was significantly associated with a decreased risk of renal impairment especially in middle and elderly-aged diabetic women.

Keywords: Coffee; Korea National Health and Nutrition Examination Surveys; Glomerular Filtration Rate; Diabetes Mellitus

Received: August 23, 2012, Accepted: May 20, 2013

*Corresponding Author: Jung Kwon Lee

Tel: +82-2-3410-2441, Fax: +82-2-3410-0388

E-mail: jklee@skku.edu

Korean Journal of Family Medicine

Copyright (C) 2013 The Korean Academy of Family Medicine

(c) This is an open-access article distributed under the terms of the Creative Commons Attribution Non-Commercial License (http://creativecommons.org/licenses/by-nc/3.0) which permits unrestricted noncommercial use, distribution, and reproduction in any medium, provided the original work is properly cited.

\section{INTRODUCTION}

Nutrition is becoming the focus of public attention due to the growing awareness of the health-promoting properties of certain foods. Fluids and beverages are an important part of our daily diet, and in particular, coffee is a major beverage in the daily lives of many Koreans. It is well known that coffee, which is second to water in terms of consumption, ${ }^{1)}$ has steadily been increasing in popularity. Approximately 500 billion cups of coffee 
are consumed annually worldwide ${ }^{2)}$ and it is estimated that adult Koreans drank, on average, 338 cups of coffee in 2011.3) Reflecting this trend of increasing coffee consumption, many studies have been conducted to examine the association between coffee and various diseases, such as diabetes, cardiovascular disease, ${ }^{4)}$ and several cancers. ${ }^{5)}$ Among such studies, an inverse association between coffee consumption and diabetes has been identified by a systematic review. ${ }^{6)}$ Although there is a paucity of knowledge regarding the relationships between coffee consumption and the risk of renal function impairment, recent small-sized Japanese studies have suggested that habitual coffee consumption may be associated with normal or increased estimated glomerular filtration rate (eGFR). ${ }^{7-9)}$ Since renal function impairment is often caused by diabetes, even in the early stage of pathogenesis, ${ }^{10)}$ one could speculate that coffee consumption may have favorable effects on kidney function via antidiabetic effects. Moreover, the beneficial effect of coffee consumption on vascular disorders in middle-aged and elderly diabetic American women has been reported to occur through coffee consumption's inverse association with markers of inflammation and endothelial dysfunction. ${ }^{11)}$ In the present study, we used large-scale and nationally representative data from the Fourth Korea National Health and Nutrition Examination Survey (KNHANES) to determine whether coffee consumption is associated with impaired renal function of Korean women with and without diabetes.

\section{METHODS}

\section{Data Collection}

KNHANES IV was conducted by the Korean Centers for Disease Control and Prevention (KCDC) between 2007 and 2009. KNHANES is a nationally representative survey as described previously. ${ }^{12)}$ The survey consisted of 4 components: the Health Interview Survey, the Health Behavior Survey, the Nutrition Survey, and the Health Examination Survey. The data were collected through standardized health examinations and household interviews with self-reported questionnaires. The health examination data consisted of height, body weight, waist circumference, body mass index (BMI), blood pressure, total cholesterol, triglyceride, high-density lipoprotein (HDL) cholesterol, and fasting blood glucose. The questionnaire included demographic, socioeconomic and lifestyle characteristics. Such as marital status, education level, household income, medical history, current drug use, nutritional habits, smoking, alcohol consumption, daily physical activity, and other health-related behaviors.

\section{Subjects}

In the 2008, survey conducted for KNHANES IV, 12,528 subjects from 4,600 households were selected using a stratified, multistage probability sampling design, and 9,744 subjects participated in the survey. As incidence of renal impairment increases with age, we included middle-aged and elderly women but excluded extremely elderly women older than 85 . Of the 3,160 women aged 35 to 84 years, 487 who did not respond to the coffee questionnaire or who lacked data about diabetes were excluded. After all selection procedures were complete, a total of 2,673 middle and elderly aged Korean women were analyzed.

\section{Assessment of Variables}

Height was measured in millimeters and body weight was measured in kilograms to the first decimal. BMI was calculated as body weight divided by height squared $\left(\mathrm{kg} / \mathrm{m}^{2}\right)$. Blood pressure was measured manually 3 times with at least 5 minutes of rest in the sitting position, with a standard mercury sphygmomanometer (Baumanometer; WA Baum Co., Inc., Copiague, NY, USA). The second and third measurements were averaged for analysis. Blood samples were obtained in the morning after at least 12 hours of midnight fasting, and total cholesterol, triglyceride, HDL cholesterol, fasting blood glucose, and creatinine were measured by enzyme techniques using an auto-analyzer (ADVIA 1650; Bayer, Tarrytown, NY, USA).

The Health Interview Survey included questions regarding whether participants were currently taking medications for hypertension, diabetes or dyslipidemia. The participants were defined as smokers if they smoked more than 5 packs during their lifetime. Likewise, alcohol drinkers were defined as those who ingested alcohol $\geq 1$ time per month according to the public health index. Alcohol Use Disorders Identification Test (AUDIT) scores were calculated and individuals were excluded if they had an AUDIT score of $\geq 8$ points. Active physical exercise was defined as at least one of the following conditions: vigorous- 
intensity physical activities of more than 20 minutes 3 times a week, moderate-intensity aerobic physical activities of more than 30 minutes on 5 times a week, or walk more than 30 minutes 5 times each week.

The eGFR was derived from the Modification of Diet in Renal Disease equation: $\mathrm{eGFR}=186.3 \times(\text { serum creatinine })^{-1.154}$ $\times(\text { age })^{-0.203}(\times 0.742$, if female $) .{ }^{13)}$ Renal function impairment was defined as eGFR $<60 \mathrm{~mL} / \mathrm{min} / 1.73 \mathrm{~m}^{2}$, according to the National Kidney Foundation classification. ${ }^{14)}$ Some studies ${ }^{15,16)}$ simply defined chronic kidney disease as eGFR $<60 \mathrm{~mL} /$ $\min / 1.73 \mathrm{~m}^{2}$. However, the actual definition of chronic kidney disease $(\mathrm{CKD})$ is based on the presence of kidney damage (i.e., albuminuria) or persistent decreased kidney function for $\geq 3$ months, as well as eGFR. ${ }^{14,17,18)}$ For this reason, it would not be proper for us to use a single measurement of eGFR $<60 \mathrm{~mL} / \mathrm{min} / 1.73 \mathrm{~m}^{2}$ as the diagnostic criterion for CKD. Instead, we used the expression of "renal function impairment." Homeostasis model of assessment-insulin resistance (HOMAIR) was calculated using the following formula: HOMA-IR = fasting insulin $(1 \mathrm{U} / \mathrm{mL}) \times$ fasting blood glucose $(\mathrm{mmol} / \mathrm{L}) / 22.5$. Diabetes was defined as a fasting blood glucose level of $\geq 126 \mathrm{mg} /$ $\mathrm{dL}$ or current treatment with hypoglycemic agents. Hypertension

Table 1. Baseline characteristics of study subjects by coffee consumption ( $n=2,673$, women aged 35 to 84 years)

\begin{tabular}{|c|c|c|c|c|}
\hline \multirow{2}{*}{ Variable } & \multicolumn{3}{|c|}{ Coffee consumption (cup/d) } & \multirow{2}{*}{ P-value* } \\
\hline & $<1$ & 1 & $\geq 2$ & \\
\hline Un-weighted no. (estimated \%) & $1,007(34.6)$ & 789 (30.6) & 877 (34.7) & $\mathrm{NA}^{\dagger}$ \\
\hline Age (y) & $56.5 \pm 0.6$ & $52.6 \pm 0.5$ & $48.6 \pm 0.4$ & $<0.001$ \\
\hline Smoker ( $\geq 5$ packs) & $8.4(1.3)$ & $7.3(1.0)$ & $9.9(1.2)$ & 0.31 \\
\hline Alcohol intake ( $\geq 1$ time/mo) & $24.4(1.7)$ & $39.1(2.0)$ & $47.8(2.0)$ & $<0.001$ \\
\hline AUDIT score ( $\geq 8$ points) & $9.2(1.4)$ & $12.7(1.5)$ & $15.6(1.6)$ & 0.01 \\
\hline Active physical activity & $53.6(2.3)$ & $53.9(2.2)$ & $54.5(2.0)$ & 0.95 \\
\hline Total energy intake $(\mathrm{kcal} / \mathrm{d})^{\ddagger}$ & $1,519.1 \pm 21.7$ & $1,586.8 \pm 23.6$ & $1,645.0 \pm 24.4$ & $<0.001$ \\
\hline \multicolumn{5}{|l|}{ Medication for } \\
\hline Diabetes & $10.5(1.1)$ & $7.7(1.0)$ & $2.3(0.6)$ & $<0.001$ \\
\hline Hypertension & $27.3(1.7)$ & $23.9(1.6)$ & $11.4(1.2)$ & $<0.001$ \\
\hline Dyslipidemia & $5.7(0.8)$ & $4.8(0.8)$ & $2.1(0.5)$ & 0.001 \\
\hline Body mass index $\left(\mathrm{kg} / \mathrm{m}^{2}\right)$ & $23.7 \pm 0.1$ & $23.7 \pm 0.2$ & $23.7 \pm 0.1$ & 1.00 \\
\hline Systolic blood pressure (mm Hg) & $118.5 \pm 0.7$ & $115.7 \pm 0.7$ & $113.6 \pm 0.8$ & $<0.001$ \\
\hline Diastolic blood pressure (mm Hg) & $75.0 \pm 0.4$ & $74.1 \pm 0.4$ & $74.2 \pm 0.4$ & 0.35 \\
\hline Fasting plasma glucose $(\mathrm{mg} / \mathrm{dL})^{\ddagger}$ & $101.5 \pm 1.1$ & $99.2 \pm 1.0$ & $96.9 \pm 0.9$ & 0.001 \\
\hline Total cholesterol $(\mathrm{mg} / \mathrm{dL})^{\ddagger}$ & $194.1 \pm 1.6$ & $191.6 \pm 1.4$ & $192.7 \pm 1.5$ & 0.99 \\
\hline Triglyceride $(\mathrm{mg} / \mathrm{dL})^{\ddagger}$ & $134.5 \pm 4.1$ & $120.0 \pm 4.5$ & $116.5 \pm 3.0$ & $<0.001$ \\
\hline HDL-cholesterol (mg/dL) & $47.7 \pm 0.4$ & $50.2 \pm 0.5$ & $50.8 \pm 0.4$ & $<0.001$ \\
\hline Creatinine (mg/dL) & $0.83 \pm 0.01$ & $0.82 \pm 0.01$ & $0.81 \pm 0.01$ & 0.13 \\
\hline Estimated GFR $\left(\mathrm{mL} / \mathrm{min} / 1.73 \mathrm{~m}^{2}\right)^{\ddagger}$ & $80.9 \pm 1.1$ & $81.7 \pm 1.1$ & $83.3 \pm 1.0$ & 0.007 \\
\hline $\mathrm{HOMA} \mathrm{IR}^{\ddagger}$ & $2.77 \pm 0.14$ & $2.55 \pm 0.10$ & $2.52 \pm 0.13$ & 0.21 \\
\hline
\end{tabular}

Values are presented as estimated mean \pm standard error or estimated percent (standard error), as appropriate.

AUDIT: Alcohol Use Disorders Identification Test, HDL: high density lipoprotein, GFR: glomerular filtration rate, HOMA-IR: homeostasis model of assessment-insulin resistance.

${ }^{*}$ Calculated using analysis of variance with Bonferroni correction or the chi-square test. ${ }^{\dagger}$ Not available. ${ }^{\star}$ Values presented are the estimated means, but log values were used for comparisons. 
was defined as a systolic blood pressure level of $\geq 140 \mathrm{~mm} \mathrm{Hg}$, a diastolic blood pressure level of $\geq 90 \mathrm{~mm} \mathrm{Hg}$, or reporting current hypertensive drug use.

\section{Coffee Consumption}

Self-reported coffee consumption data were obtained through dietary interviews. Participants were asked how many cups of coffee they drank during a day, a week or a month. In the questionnaire, the frequency of coffee intake was classified into the ten categories: rarely, 6-11 cup/y, $1 \mathrm{cup} / \mathrm{mo}, 2-3 \mathrm{cup} / \mathrm{mo}, 1 \mathrm{cup} / \mathrm{wk}, 2-3 \mathrm{cup} /$ wk, 4-6 cup/wk, 1 cup/d, 2 cup/d, and $\geq 3$ cup/d. Frequency of coffee consumption was assorted into 3 groups ( $<1$ cup/d, 1 cup/d, and $\geq 2 \mathrm{cup} / \mathrm{d}$ ) because of the small difference between the individual participants and the excessively small samples. No distinction was made between caffeinated and decaffeinated coffee, or between the individual types of coffee (boiled, filtered, and instant).

\section{Statistical Analysis}

All statistical analyses were conducted with SPSS ver. 18.0 (SPSS Inc., Chicago, IL, USA). Statistical significance was defined as a $\mathrm{P}$-value of $<0.05$. All estimates were calculated using sample weights to represent the entire Korean population and adjusted for the complex sample design of the survey. Odds ratios (ORs) and 95\% confidence intervals (CIs) for renal function impairment-associated coffee consumption were estimated with a multiple logistic regression model. The baseline factors were adjusted for age, alcohol intake, total energy intake, hypertension, antidyslipidemic drug use, and BMI. To improve the normality distribution, the values of total energy intake, fasting plasma glucose, total cholesterol, triglycerides, eGFR, and HOMAIR were $\log$ transformed. Persons who drank $<1$ cup of coffee per day were used as a reference group. Stratified analyses were performed based on the absence or presence of diabetes to establish whether an effect on eGFR might account for the particular effects of coffee consumption on diabetes.

\section{RESULTS}

Coffee consumption at baseline was associated with several lifestyle and metabolic factors (Table 1). Coffee drinkers were more likely to be younger, consume alcohol $\geq 1$ time per

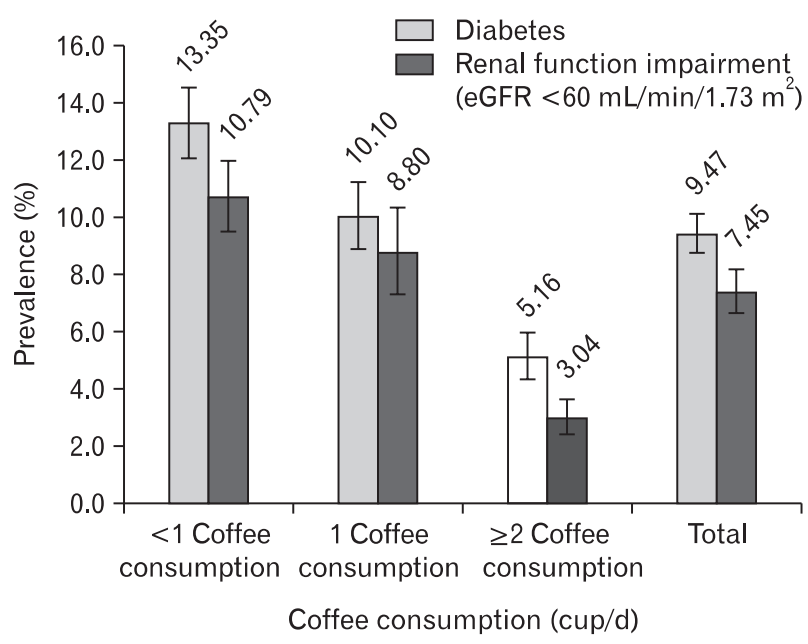

Figure 1. Prevalence of diabetes and renal function impairment (eGFR $<60 \mathrm{~mL} / \mathrm{min} / 1.73 \mathrm{~m}^{2}$ ) by coffee consumption in Korean women (All $\mathrm{P}<0.001$ by the chi-square test). eGFR: estimated glomerular filtration rate.

Table 2. Odds ratios and 95\% confidence intervals of renal function impairment (estimated glomerular filtration rate < 60) by coffee consumption in Korean women

\begin{tabular}{cccccc}
\hline & $<1$ cup/d & 1 cup/d & P-value & $\geq 2$ cup/d & P-value \\
\hline Model 1* & 1 (Reference) & $0.80(0.53-1.21)$ & 0.29 & $0.26(0.16-0.41)$ & $<0.001$ \\
Model 2 $^{\dagger}$ & 1 (Reference) & $1.13(0.74-1.73)$ & 0.56 & $0.57(0.36-0.90)$ & 0.02 \\
Model $^{\ddagger}$ & 1 (Reference) & $1.15(0.75-1.75)$ & 0.52 & $0.57(0.35-0.91)$ & 0.02 \\
Model $^{\S}$ & 1 (Reference) & $1.16(0.76-1.78)$ & 0.49 & $0.59(0.37-0.95)$ & 0.03 \\
\hline
\end{tabular}

Values are presented as odds ratios (95\% confidence intervals).

*Not adjusted. ${ }^{\dagger}$ Adjusted for age. ${ }^{\ddagger}$ Adjusted for alcohol intake, total energy intake (log transformed), hypertension, antidyslipidemic drug use, and body mass index, in addition to the covariates included in the model $2 .{ }^{\S}$ Adjusted for diabetes, in addition to the covariates included in the model 3. 
month and ingest more calories. As compared with persons who consume less coffee, more subjects who consumed coffee $\geq 1$ cup of coffee per day were less likely to use hypertensive, antidyslipidemic, or antidiabetic drugs, and to have lower levels of systolic blood pressure and higher levels of HDL. There were also significant differences in baseline eGFR between the individual groups according to increasing frequencies of coffee consumption.

The prevalence of diabetes and renal function impairment was higher in women who drank $<1$ cup of coffee per day. The prevalence of diabetes gradually decreased with increasing frequency of coffee consumption (Figure 1). The prevalence of renal function impairment $\left(\mathrm{eGFR}<60 \mathrm{~mL} / \mathrm{min} / 1.73 \mathrm{~m}^{2}\right)$ also decreased with increasing frequency of coffee consumption.

Compared with drinking $<1$ cup of coffee per day, the OR for renal function impairment was significantly lower (OR, 0.26; $95 \% \mathrm{CI}, 0.16$ to $0.41 ; \mathrm{P}<0.001)$ in those who habitually drank $\geq 2$ cups of coffee per day in non-adjusted analysis (Table 2 ). The results remained the same after adjustment for multiple confounding factors, such as age, alcohol intake, total energy intake, hypertension, antidyslipidemic drug use, and BMI (OR, 0.52 ; $95 \% \mathrm{CI}, 0.35$ to $0.91 ; \mathrm{P}=0.02$ ).

When data were stratified according to the presence of diabetes (Table 3 ), coffee consumption of $>2$ cups of coffee per day showed an inverse association with renal function impairment in only diabetic women (OR, 0.14; $95 \% \mathrm{CI}, 0.02$ to 0.88 ; $\mathrm{P}=$ 0.04 ), compared with consumption of $<1$ cup of coffee per day. There was no significant association between coffee consumption and renal function impairment in women without diabetes.

\section{DISCUSSION}

In this large nationwide representative study, we observed that habitual coffee consumption of $\geq 2$ cups per day was significantly associated with a decreased risk of renal function impairment as compared with coffee consumption of $<1$ cup per day in women with diabetes. It was the first study to examine the association between coffee consumption and renal function impairment in Korean women. Several studies in Japan have reported the effects of coffee on kidney function. In a cross-sectional study of 342 healthy adults, drinking $\geq 1$ cup of coffee per day was significantly associated with normal or increased eGFR $(\geq 90 \mathrm{~mL} / \mathrm{min} / 1.73$ $\left.\mathrm{m}^{2}\right)$. $^{7)}$ Another cross-sectional study of 114 community-based health checkup subjects has also reported that the consumption of $\geq 1$ cup of coffee per day was associated with higher eGFR as compared with non-coffee drinkers. ${ }^{8)}$ The results were significant and independent of the added sugar and level of high sensitivity

Table 3. ORs and 95\% CIs of renal function impairment (estimated glomerular filtration rate < 60) by coffee consumption in Korean women with or without diabetes

\begin{tabular}{|c|c|c|c|c|c|c|c|}
\hline & \multirow{2}{*}{ Prevalence \% (SE) } & \multicolumn{3}{|c|}{ Model 1* } & \multicolumn{3}{|c|}{ Model $2^{\dagger}$} \\
\hline & & OR (95\% CI) & P-value & P for trend & OR (95\% CI) & P-value & P for trend \\
\hline With diabetes (cup/d) & & & & 0.05 & & & 0.03 \\
\hline$<1$ & $24.1(4.4)$ & 1 (Reference) & & & 1 (Reference) & & \\
\hline 1 & $22.8(5.2)$ & $1.13(0.52-2.44)$ & 0.75 & & $1.02(0.48-2.17)$ & 0.96 & \\
\hline$\geq 2$ & $3.2(2.3)$ & $0.16(0.04-0.75)$ & 0.02 & & $0.14(0.02-0.88)$ & 0.04 & \\
\hline Without diabetes (cup/d) & & & & 0.24 & & & 0.21 \\
\hline$<1$ & $8.7(1.2)$ & 1 (Reference) & & & 1 (Reference) & & \\
\hline 1 & $7.2(1.5)$ & $1.15(0.70-1.88)$ & 0.58 & & $1.16(0.71-1.91)$ & 0.55 & \\
\hline$\geq 2$ & $3.0(0.6)$ & $0.69(0.43-1.13)$ & 0.14 & & $0.67(0.41-1.11)$ & 0.12 & \\
\hline
\end{tabular}

Values were presented as odds ratios (95\% confidence intervals).

OR: odds ratio, CI: confidence interval, SE: standard error.

*Adjusted for age. ${ }^{\dagger}$ Adjusted for alcohol intake, total energy intake (log transformed), body mass index, hypertension, homeostasis model of assessment-insulin resistance (log transformed) and antidyslipidemic drug use in addition to the covariates included in the model 1. 
C-reactive protein. One clinical trial of 19 young adults aged 21 to 27 years also showed that the consumption of $\geq 3$ cups of coffee per day for 14 consecutive days is significantly associated with increased cystatin C-based eGFR as compared with subjects who consume green tea consumption. ${ }^{9)}$ This finding suggests that even a short period of coffee consumption may have a favorable effect on kidney function. However, these aforementioned studies were conducted with only a few participants and did not consider the impact of diabetes on the association between coffee consumption and kidney function.

Several plausible explanations for our results are as follows. First, the various components of coffee may protect the glomerular endothelium from oxidative stress. Caffeic acid, hydroxyhydroquinone, and chlorogenic acid are known antioxidants contained in coffee. ${ }^{19)}$ Second, the antidiabetic effect of coffee may decrease development of diabetic nephropathy. Since phenol chlorogenic acid and its degradation products inhibit glucose absorption and hepatic glucose output, it is conceivable that higher coffee intake may be related to a decreased risk of diabetes. ${ }^{20)}$ Reverse causality is also a possible explanation. Persons with chronic disease, such as diabetes and chronic kidney disease, might avoid coffee drinking.

The prevalence of diabetes decreased as coffee consumption increased. Our results are concordant with those of previous studies showing an inverse association between coffee consumption and diabetes. ${ }^{6)}$ The prevalence of renal function impairment also decreased gradually with the increasing frequency of coffee consumption. Although data were not shown, there was no significant association in middle and elderly-aged Korean men and the mechanism is not clear. Further studies are needed.

There are some limitations to our study. Since coffee consumption was assessed only at the baseline, our results may not reflect long-term patterns of consumption. In our study, there was a lack of information about the type of coffee (caffeinated or decaffeinated), the contents of coffee consumed, whether sugar or cream was added and how the coffee was prepared (espresso, boiled, or filtered), and this may have affected the results. Also, as the categories of coffee consumption were broad $(<1 \mathrm{cup} / \mathrm{d}$, 1 cup/d, and $\geq 2$ cup/d), they may not have accurately reflected the dose-dependent effects of coffee consumption. Given the observational nature of our study, the inverse relationship between coffee consumption and renal function impairment may not reflect a cause-and-effect relationship. And the association may not be definitive due to unmeasured or poorly measured confounders.

Nevertheless, our study has some strengths. First, the sample size of our study was larger than those of prior studies and was based on KNHNES data, which ensured reliable country-wide sampling. Second, more variant multiple confounding factors were adjusted for a variety of parameters compared with previous studies and the results remained significant after the adjustment.

In conclusion, this large cross-sectional study revealed an inverse association between coffee consumption and decreased eGFR, especially in middle-aged and elderly diabetic women. Our results suggest that coffee consumption may be associated with a lower risk of diabetes development, a decreased risk of renal impairment in patients who have already developed diabetes, and a reduction in diabetic nephropathy. Further randomized, controlled clinical studies are needed to confirm the effects of coffee on kidney function.

\section{CONFLICT OF INTEREST}

No potential conflict of interest relevant to this article was reported.

\section{REFERENCES}

1. Prakash NS, Combes MC, Somanna N, Lashermes P. AFLP analysis of introgression in coffee cultivars (Coffea arabica L.) derived from a natural interspecific hybrid. Euphytica 2002; 124:265-71.

2. Clarke RJ, Vitzthum OG. Coffee: recent developments. Oxford: Blackwell Science; 2001.

3. Korea customs service. Coffee income trends [Internet]. Daejeon: Korea customs service; 2012 [cited 2012 Jun 27]. Available from: http://www.customs.go.kr/.

4. Mesas AE, Leon-Munoz LM, Rodriguez-Artalejo F, Lopez-Garcia E. The effect of coffee on blood pressure and cardiovascular disease in hypertensive individuals: a systematic review and meta-analysis. Am J Clin Nutr 2011; 
94:1113-26.

5. Arab L. Epidemiologic evidence on coffee and cancer. Nutr Cancer 2010;62:271-83.

6. Van Dam RM, Hu FB. Coffee consumption and risk of type 2 diabetes: a systematic review. JAMA 2005;294:97-104.

7. Nakajima K, Hirose K, Ebata M, Morita K, Munakata H. Association between habitual coffee consumption and normal or increased estimated glomerular filtration rate in apparently healthy adults. Br J Nutr 2010;103:149-52.

8. Kotani K, Sakane N, Yamada T, Taniguchi N. Association between coffee consumption and the estimated glomerular filtration rate in the general Japanese population: preliminary data regarding C-reactive protein concentrations. Clin Chem Lab Med 2010;48:1773-6.

9. Saito M, Nemoto T, Tobimatsu S, Ebata M, Le Y, Nakajima K. Coffee consumption and cystatin-C-based estimated glomerular filtration rates in healthy young adults: results of a clinical trial.J Nutr Metab 2011;2011:146865.

10. Kramer H. Screening for kidney disease in adults with diabetes and prediabetes. Curr Opin Nephrol Hypertens 2005;14:249-52.

11. Lopez-Garcia E, van Dam RM, Qi L, Hu FB. Coffee consumption and markers of inflammation and endothelial dysfunction in healthy and diabetic women. Am J Clin Nutr 2006;84:888-93.

12. Park YS, Kim JS. Obesity phenotype and coronary heart disease risk as estimated by the Framingham risk score. J Korean Med Sci 2012;27:243-9.
13. Levey AS, Bosch JP, Lewis JB, Greene T, Rogers N, Roth D. A more accurate method to estimate glomerular filtration rate from serum creatinine: a new prediction equation. Modification of Diet in Renal Disease Study Group. Ann Intern Med 1999;130:461-70.

14. National Kidney Foundation. K/DOQI clinical practice guidelines for chronic kidney disease: evaluation, classification, and stratification. Am J Kidney Dis 2002;39(2 Suppl 1):S1-266.

15. Chen J, Muntner P, Hamm LL, Fonseca V, Batuman V, Whelton PK, et al. Insulin resistance and risk of chronic kidney disease in nondiabetic US adults. J Am Soc Nephrol 2003;14:469-77.

16. Chen J, Muntner P, Hamm LL, Jones DW, Batuman V, Fonseca $\mathrm{V}$, et al. The metabolic syndrome and chronic kidney disease in U.S. adults. Ann Intern Med 2004;140:167-74.

17. Vassalotti JA, Stevens LA, Levey AS. Testing for chronic kidney disease: a position statement from the National Kidney Foundation. Am J Kidney Dis 2007;50:169-80.

18. Stevens LA, Levey AS. Current status and future perspectives for CKD testing. Am J Kidney Dis 2009;53(3 Suppl 3):S1726.

19. Butt MS, Sultan MT. Coffee and its consumption: benefits and risks. Crit Rev Food Sci Nutr 2011;51:363-73.

20. Zhang Y, Lee ET, Cowan LD, Fabsitz RR, Howard BV. Coffee consumption and the incidence of type 2 diabetes in men and women with normal glucose tolerance: the Strong Heart Study. Nutr Metab Cardiovasc Dis 2011;21:418-23. 